\title{
ALIMENTAÇÃO E CICLO ALIMENTAR DIÁRIO DE HOPLOSTERNUM LITTORALE (HANCOCK) (SILURIFORMES, CALLICHTHYIDAE) NAS LAGOAS GUARANÁ E PATOS DA PLANÍCIE DO ALTO RIO PARANÁ, BRASIL
}

\author{
Norma Segatti Hahn 1,2 \\ Vera Lúcia Lescano de Almeida ${ }^{1,3}$ \\ Karla Danielle Gaspar da Luz ${ }^{4}$
}

\begin{abstract}
FEEDING AND DIEL CYCLE OF FEEDING OF HOPLOSTERNUM LITTORALE (HanCock) (Siluriformes, CAllichthyidae) in THE lagoons Guaraná and Patos OF THE UPPER Parana RIVER, BrazIL. Hoplosternum littorale (Hancock, 1828) is an abundant species in the lagoons of the upper Parana river floodplain. This species was sampled in Patos and Guarana lagoons in the flood period (November/92-May/93) and low water period (June-October/93). To analyse food contents in the diet were analysed stomach of 91 adults. Twenty seven food items were recorded, among them, Testaceae, Nematoda, microcrustaceans, quironomids and organic detritus were predominant. These items allows to characterize the species as bentivore. To study feeding activity were analized 565 adults stomach contents. Food intake was more intense during flooding. Considering diel variation, the species started to eat during dusk, increasing during night, stopping to eat during daytime.

KEY WORDS. Siluriformes, Callichthyidae, Hoplosternum littorale, feeding, diel cycle, lagoons
\end{abstract}

A planície de inundação do alto rio Paraná está inserida no último trecho não represado desse rio em território brasileiro. Esse ambiente, embora sujeito a grandes variações sazonais e espaciais na composição ictíica, apresenta uma rica assembléia de peixes, com cerca de 170 espécies identificadas (AGOSTINHO \& ZALEWSKI 1996). Entre elas, o tamboatá Hoplosternum littorale (Hancock, 1828), um siluriforme, predominante nas lagoas, que são ambientes rasos e ricos em macrófitas aquáticas. Essa espécie, além de possuir um alto valor comercial em alguns países da América do Sul, apresenta como cacterísticas, fácil reprodução em ambientes confinados e boa tolerância a baixos níveis de oxigênio, o que a torna propícia ao cultivo (LUQUET et al. 1990).

A despeito dos inúmeros estudos sobre a ictiofauna do rio Paraná, não existe registro sobre a biologia do tamboatá. A alimentação e a reprodução dessa espécie

1) Departamento de Biologia, Nupélia, Universidade Estadual de Maringá. Avenida Colombo 5790, 87020-900 Maringá, Paraná, Brasil.

2) Pesquisador do CNPq.

3) Curso de Pós-graduação em Ambientes Aquáticos Continentais.

4) Bolsista de Iniciação Cientifica RHAE/CNPq. 
foram descritas por WINEMILLER (1987) em Cano Maraca na planície de inundação da Venezuela, e estudos experimentais sobre atividade alimentar têm sido desenvolvidos por BOUJARD et al. $(1990,1991,1992)$.

Neste trabalho são descritas a alimentação e o ciclo diário de ingestão de alimento de exemplares de $H$. littorale capturados em duas lagoas permanentes (Guaraná e Patos), localizadas na planície de inundação do alto rio Paraná.

\section{MATERIAL E MÉTODOS}

A região de estudo inclui as lagoas Guaraná e Patos, trecho que compreende a margem direita do rio Paraná, especificamente os segmentos inferiores dos rios Baia e Ivinheima, respectivamente (Fig. 1). A lagoa do Guaraná $\left(53^{\circ} 20^{\prime} \mathrm{W}, 22^{\circ} 42^{\prime} \mathrm{S}\right)$ situa-se à margem direita do rio Baia, interligando-se a esse rio por um estreito canal. A lagoa dos Patos $\left(22^{\circ} 43^{\prime} 12 \mathrm{~S}, 53^{\circ} 17^{\prime} 37 \mathrm{~W}\right)$ localiza-se à margem direita do rio Paraná e a margem esquerda do rio Ivinheima, com o qual se comunica através de um canal durante $o$ ano todo.

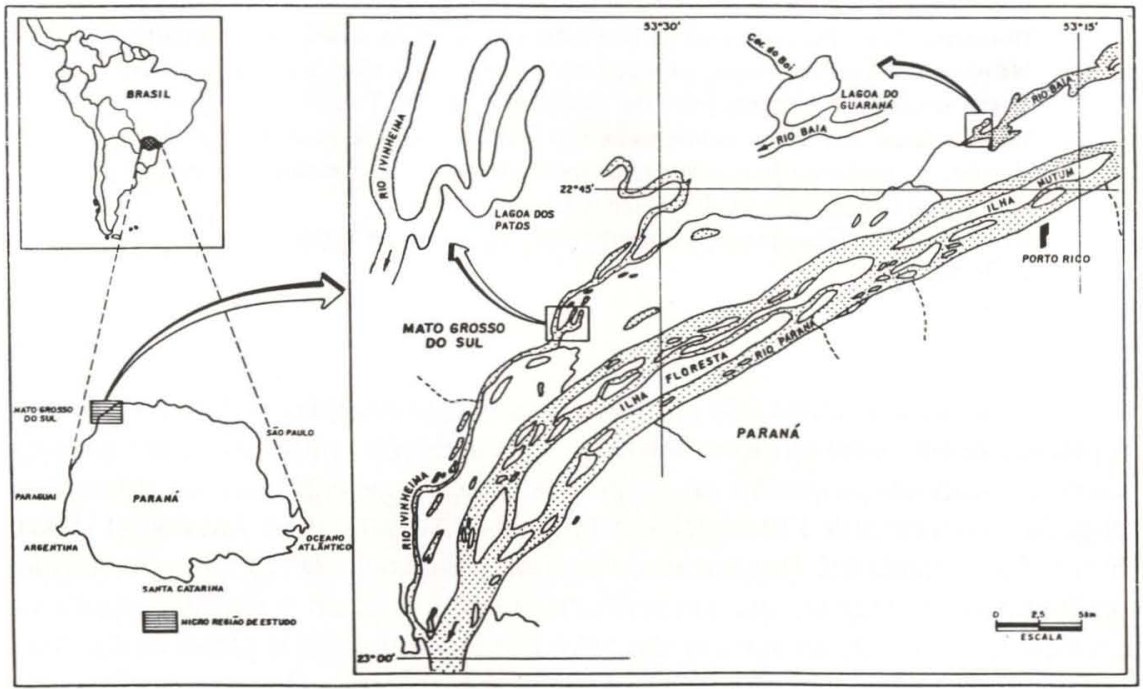

Fig. 1. Localização geográfica da região de estudo com indicação dos locais de coleta.

As coletas foram realizadas nos períodos de cheia (março, abril, maio, novembro, dezembro/92 e janeiro, fevereiro/93) e seca (junho a outubro/92) (Fig. 2). Para as capturas dos peixes, foram utilizadas redes de espera simples e tresmalho de diferentes malhagens ( $3-16 \mathrm{~cm}$ ), que ficaram expostas por 24 horas, com despescas a cada quatro horas, em cada lagoa. Após a despesca e obtenção de dados biométricos de rotina (Ls, Wt), o material biológico para análise foi fixado em formol $4 \%$. 


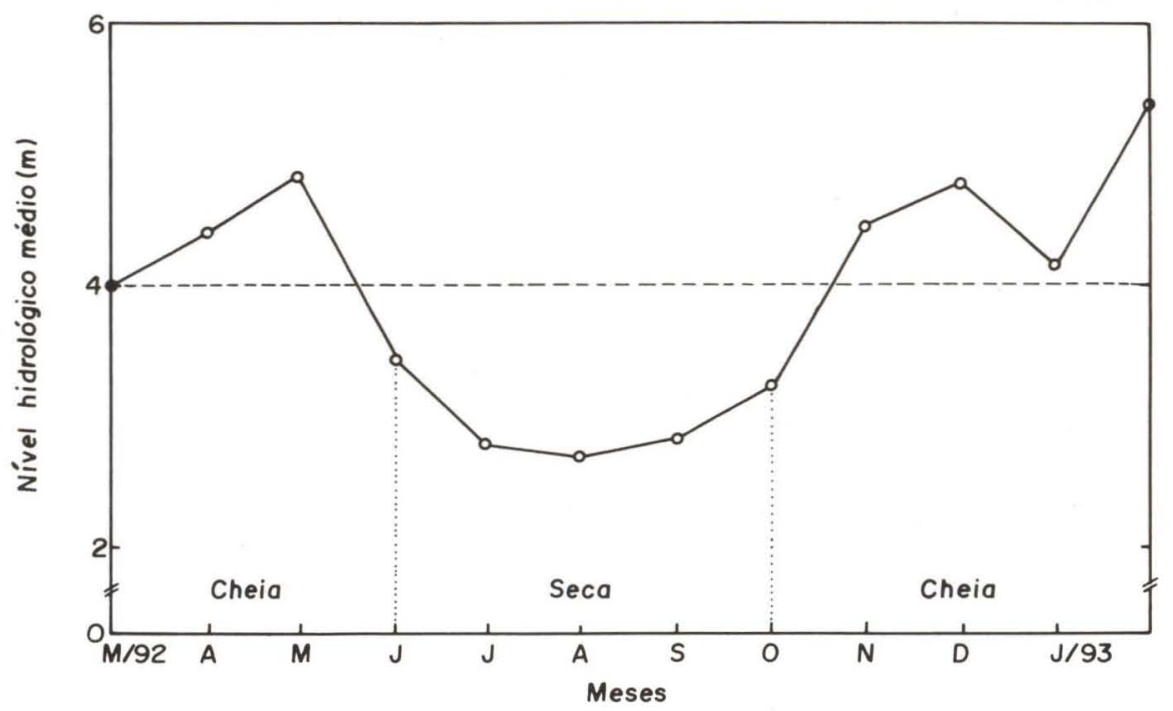

Fig. 2. Variação mensal do nivel hidrológico do rio Paraná de março/92 a fevereiro/93 com indicação das fases de cheia e seca.

Para a determinação da dieta, foram analisados os conteúdos estomacais de 91 exemplares adultos $(\mathrm{Ls}=8,3-18,5 \mathrm{~cm})$ através dos métodos de freqüência de ocorrência e pontos (HYSLOP 1980), que foram combinados no Índice Alimentar (IAi) (KAWAKAMI \& VAZZOLER 1980), e expressos em porcentagem. Para caracterização de jovens e adultos, considerou-se o tamanho de primeira maturação de acordo com VAZZOLER (1996). No entanto, o número insuficiente de jovens não permitiu incluí-los nas análises da dieta e da atividade alimentar.

Para a atividade alimentar, foram examinados os conteúdos estomacais de 565 indivíduos adultos, a qual foi avaliada através do grau médio de repleção estomacal, em cada horário de captura, de acordo com a seguinte escala: (0) estômago vazio; (1) parcialmente vazio (volume do estômago ocupado até $30 \%$ ); (2) parcialmente cheio (volume ocupado de $30 \%$ a $60 \%$ ) e (3) cheio (volume ocupado acima de $60 \%$ ).

\section{RESULTADOS}

Os resultados da análise de conteúdos estomacais de indivíduos adultos de H. littorale, mostraram um espectro amplo e variado de tipos de alimento (27 itens), embora poucos tenham sido dominantes, na dieta, em ambas as lagoas (Tabs I, II). De modo geral, os grupos mais explorados foram os microcrustáceos (Cladocera e Ostracoda), representando cerca de $20 \%$ do IAi, quironomídeos (cerca de $20 \%$ ) e nematóides (cerca de 10\%). Além desses, detrito orgânico e tecamebas destacaramse também na alimentação dos exemplares capturados na lagoa do Guaraná, ambos representando cerca de $12 \%$ do IAi. A dieta de $H$. littorale na lagoa do Guaraná e na lagoa dos Patos foi significativamente similar (SPEARMAN rs=0,833; $\mathrm{P}=0,01$ ). 
Tabela I. Freqüência de ocorrência, volumétrica e do Indice Alimentar (IAi) dos itens encontrados nos conteúdos gástricos de adultos de $\mathrm{H}$. littorale da lagoa dos Patos $(\mathrm{N}=37)$.

\begin{tabular}{|c|c|c|c|c|c|}
\hline Itens & Ocorrência & $(\%)$ & Volume & $(\%)$ & $\mid \mathrm{Ai}(\%)$ \\
\hline Tecameba & 24 & 9,45 & 47 & 5,97 & 7,84 \\
\hline Nematoda & 23 & 9,06 & 62 & 7,88 & 9,93 \\
\hline Rotifero & 4 & 1,57 & 22 & 2,80 & 0,61 \\
\hline Bryozoa & 11 & 4,33 & 22 & 2,80 & 1,69 \\
\hline Acarina & 10 & 3,94 & 25 & 3,18 & 1,74 \\
\hline Bivalvia & 3 & 1,18 & 15 & 1,90 & 0,31 \\
\hline Gastropoda & 9 & 3,54 & 28 & 3,56 & 1,75 \\
\hline Cladocera & 25 & 9,84 & 75 & 9,53 & 13,04 \\
\hline Copepoda & 15 & 5,91 & 37 & 4,70 & 3,86 \\
\hline Conchostraca & 4 & 1,57 & 17 & 2,16 & 0,47 \\
\hline Ostracoda & 33 & 12,99 & 106 & 13,47 & 24,31 \\
\hline Chironomidae (larva) & 31 & 12,21 & 98 & 12,45 & 21,12 \\
\hline Coleoptera (adulto) & 3 & 1,18 & 10 & 1,27 & 0,21 \\
\hline Coleoptera (larva) & 2 & 0,79 & 4 & 0,51 & 0,06 \\
\hline Ephemeroptera (larva) & 3 & 1,18 & 23 & 2,92 & 0,48 \\
\hline Hemiptera (adulto) & 4 & 1,57 & 23 & 2,92 & 0,64 \\
\hline Odonata (larva) & 2 & 0,79 & 17 & 2,16 & 0,24 \\
\hline Orthoptera (adulto) & 1 & 0,39 & 2 & 0,25 & 0,01 \\
\hline Resto de inseto & 8 & 3,15 & 32 & 4,07 & 1,78 \\
\hline Escamas & 4 & 1,57 & 9 & 1,14 & 0,25 \\
\hline Algas & 5 & 1,97 & 7 & 0,89 & 0,24 \\
\hline Detrito vegetal & 7 & 2,76 & 25 & 3,18 & 1,22 \\
\hline Detrito orgânico & 16 & 6,30 & 68 & 8,64 & 7,57 \\
\hline Grão de areia & 7 & 2,76 & 13 & 1,65 & 0,63 \\
\hline
\end{tabular}

Do total de indivíduos capturados, 57,3\% encontravam-se com estômagos vazios. A atividade alimentar diária, em cada lagoa e fase do ciclo hidrológico, está representada na figura 3. Em ambas as lagoas, a tomada de alimento foi mais acentuada durante a cheia.

Com relação a atividade alimentar diária, verifica-se que durante a cheia os padrões de tomada de alimento são mais nítidos independente de variações sazonais ou espaciais, a alimentação é mais intensa nos horários crepusculares, intensificando-se durante à noite, e diminuindo ou até cessando durante o dia. Observa-se, inclusive, ausência de captura durante o dia, no período de águas baixas, principalmente na lagoa dos Patos.

\section{DISCUSSÃo}

A morfologia funcional de $H$. littorale (boca sub-inferior, corpo coberto por placas ósseas e achatado ventralmente) permite inferir que se trata de uma espécie pouco ágil e que habita o fundo dos corpos de água. Aliado a isto, o tipo de alimento consumido, ou seja, organismos da infauna bentônica, associado a detritos e grãos de areia, permite caracterizá-la como espécie bentófaga. 
Tabela II. Freqüência de ocorrência, volumétrica e do Índice Alimentar (IAi) dos itens encontrados nos conteúdos gástricos de adultos de $H$. littorale da lagoa do Guaraná $(\mathrm{N}=54)$.

\begin{tabular}{|c|c|c|c|c|c|}
\hline Itens & Ocorrência & $(\%)$ & Volume & $(\%)$ & $\mathrm{IAi}(\%)$ \\
\hline Tecameba & 46 & 11,41 & 125 & 9,13 & 11,97 \\
\hline Nematoda & 40 & 9,93 & 131 & 9,57 & 10,92 \\
\hline Rotifero & 1 & 0,25 & 3 & 0,22 & 0,01 \\
\hline Bryozoa & 25 & 6,21 & 48 & 3,50 & 2,50 \\
\hline Acarina & 15 & 3,72 & 30 & 2,19 & 0,94 \\
\hline Bivalvia & 1 & 0,25 & 2 & 0,15 & 0,01 \\
\hline Gastropoda & 12 & 2,98 & 45 & 3,29 & 1,12 \\
\hline Cladocera & 46 & 11,41 & 190 & 13,88 & 18,20 \\
\hline Copepoda & 34 & 8,44 & 118 & 8,62 & 8,36 \\
\hline Conchostraca & 8 & 1,99 & 2 & 0,15 & 0,03 \\
\hline Ostracoda & 37 & 9,18 & 127 & 9,28 & 9,79 \\
\hline Chironomidae (larva) & 52 & 12,90 & 201 & 14,68 & 21,77 \\
\hline Coleoptera (larva) & 2 & 0,50 & 9 & 0,66 & 0,04 \\
\hline Ephemeroptera (adulto) & 4 & 0,99 & 12 & 0,88 & 0,10 \\
\hline Ephemeroptera (larva) & 2 & 0,50 & 12 & 0,88 & 0,05 \\
\hline Hemiptera (adulto) & 3 & 0,74 & 16 & 1,17 & 0,10 \\
\hline Homoptera (adulto) & 1 & 0,25 & 2 & 0,15 & 0,01 \\
\hline Hymenoptera (adulto) & 1 & 0,25 & 4 & 0,29 & 0,01 \\
\hline Odonata (larva) & 2 & 0,50 & 7 & 0,51 & 0,20 \\
\hline Orthoptera (adulto) & 1 & 0,25 & 3 & 0,22 & 0,01 \\
\hline Trichoptera (larva) & 3 & 0,74 & 11 & 0,80 & 0,07 \\
\hline Resto de inseto & 9 & 2,23 & 44 & 3,21 & 0,82 \\
\hline Escamas & 3 & 0,74 & 8 & 0,58 & 0,05 \\
\hline Algas & 9 & 2,23 & 19 & 1,39 & 0,36 \\
\hline Detrito vegetal & 5 & 1,24 & 15 & 1,09 & 0,16 \\
\hline Detrito orgânico & 35 & 8,68 & 170 & 12,42 & 12,39 \\
\hline Gräo de areia & 6 & 1,49 & 15 & 1,09 & 0,19 \\
\hline
\end{tabular}
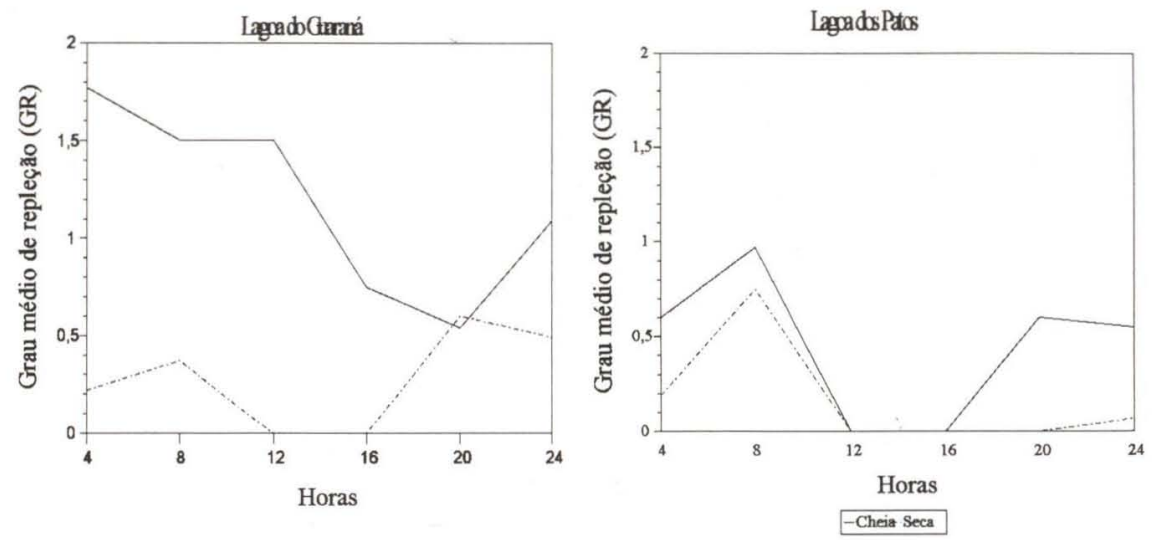

Figura 3. Variação diária do grau médio de repleção estomacal para os adultos de $H$. littorale por local e fase do ciclo hidrológico. 
Nos lhanos da Venezuela, WINEMILLER (1987) registrou para essa espécie, os mesmos grupos-tróficos, embora o autor tenha constatado diferenças na dieta quando os peixes imaturos foram comparados com os adultos, e quando os adultos foram comparados entre diferentes estações do ano. Neste estudo, análises comparativas entre jovens e adultos não foram possíveis, em função do número reduzido de estômagos com alimento para os juvenis. Por outro lado, quando comparadas as dietas dos peixes entre as duas lagoas, estas foram significativamente similares quanto a qualidade dos itens ingeridos notando-se, contudo, diferenças em suas proporções.

O maior consumo de detrito orgânico por peixes da lagoa do Guaraná pode ser explicado pelo fato de esta lagoa apresentar uma coloração caracteristicamente escura. Isto é devido à grande quantidade de compostos húmicos presentes em suas águas, decorrentes da matéria orgânica autóctone e, particularmente, alóctone, que é carreada durante as fases de inundação, acumulando-se no fundo e proporcionando, portanto, maior disponibilidade desse material (TOMAZ et al. 1992).

Os quironomídeos, muito importantes na dieta de um modo geral, devem ser tomados não só no substrato como também em raízes de macrófitas aquáticas, que são abundantes nas lagoas e onde estas larvas de insetos ocorrem em elevadas densidades (FUEM-CIAMB/PADCT 1995).

Microcrustáceos e nematóides, que também se destacaram como alimento, devem ser muito numerosos e disponíveis nestes ambientes. Os primeiros, representados principalmente por cladóceros, tiveram sua dominância constatada em ambientes lênticos da planície (LANSAC TôHA et al. 1993). Por vezes, os conteúdos estomacais continham unicamente esses organismos, sem vestígio algum de detrito ou sedimento.

Acredita-se que esses itens devem ser alterados em sua ordem de importância, conforme a oferta do ambiente, como demonstrado nessa planície por TAKEDA et al. (1990) e LANSAC TÔHA et al. (1993) para o zoobentos e zooplâncton, respectivamente. Nesse sentido, WoOTTON (1990) relata que peixes são bons amostradores e que os conteúdos gástricos refletem a disponibilidade de alimento no ambiente.

A maior atividade alimentar dos peixes na cheia pode ser explicada porque, em geral durante as chuvas ocorre um crescimento explosivo de microorganismos, devido a grande quantidade de nutrientes carreada, e também uma ampliação dos biótopos, o que conseqüentemente aumenta a disponibilidade de muitos grupos-tróficos. WINEMILLER (1987) relata que a maior proporção de indivíduos com estômagos vazios, no período seco, ocorre em função da depleção de oxigênio no ambiente aquático, o que leva $H$. littorale a cessar sua alimentação e utilizar seu intestino para a respiração aérea.

O ciclo de atividade alimentar, crepuscular-noturno, de H. littorale é evidenciado pela captura de exemplares com estômagos totalmente vazios e mesmo ausência de captura de exemplares durante o dia. Isto indica que os peixes não se encontram em atividade nas duas lagoas nas horas claras do dia, o que pode ser uma estratégia para evitar a predação por grandes piscívoros. BOUJARD et al. (1990), em 
experimento realizado com essa espécie, verificaram que a tomada voluntária do alimento também é cíclica, com uma fase acentuada de atividade alimentar entre 2:00 e 5:00 horas, ou seja, de sete a dez horas após o anoitecer natural. Esses autores também detectaram que a alternância de claro-escuro constitui o sincronizador das atividades locomotora, alimentar e respiração aérea para essa espécie. Tais resultados evidenciam que $H$. littorale é uma espécie noturna, cuja atividade alimentar apresenta um ritmo exógeno, sincronizado por um estímulo periódico externo, a luz (BOUJARD et al. 1991).

AGRADECIMENTOS. Agradecemos ao CIAMB/PADCT pelo apoio financeiro e ao NUPELIA (Núcleo de Pesquisas em Limnologia, Ictiologia e Aquicultura) pelo apoio logístico.

\section{REFERÊNCIAS BIBLIOGRÁFICAS}

Agostinho, A.A. \& M. ZALEWSKI. 1996. Upper Paraná River Floodplain: Importance and Preservation. Maringá, EDUEM, 100p.

BoujaRD, T.; P. KeITH \& P. LUQUeT. 1990. Diel cycle in Hoplosternum littorale

(Teleostei): evidence for synchronization of locomotor, air breathing and feeding activity by circadian alternation of light and dark. J. Fish Biol. 36: 133-140.

BOUJARD, T.; Y. MOREAU \& P. LUQUET. 1991. Entrainment of the circadian rhythm of food demand by infradian cycles of light-dark alternation in Hoplosternum littorale (Teleostei). Aquat. Living Resour. 4: 221-225.

1992. Diel cycle in Hoplosternum littorale (Teleostei): entrainment of feeding activity by low intensity colored light. Env. Biol. of Fishes. 35 (3): 301-309.

FUEM-CIAMB/PADCT. 1995. Relatório anual do projeto "Estudos ambientais da planície de inundação do rio Paraná, no trecho compreendido entre a foz do rio Paranapanema e o reservatório de Itaipu". Maringá, Universidade Estadual de Maringá, Vol. 2, 459p.

HYSLOP, E.J. 1980. Stomach contents analysis: a review of methods and their application. J. Fish Biol. 17 (4): 411-429.

KaWAKAmi, E. \& G. VAZzoler. 1980. Método gráfico e estimativa de indice alimentar aplicado no estudo de alimentação de peixes. Bol. Inst. Oceanogr. 29 (2): 205-207.

LansaC TôHa, F.A.; A.F. Lima; S.M. Thomaz \& M.C. Roberto. 1993. Zooplâncton de uma planície de inundação do rio Paraná. II. Variação sazonal e influência dos níveis fluviométricos sobre a comunidade. Acta Limnol. Brasil. 6: 42-55.

Luquet, P., T. Boujard; P. Planguette; Y. Moreau \& G. Hostache. 1990. The culture of Hoplosternum littorale: State of the art and perspectives, p.511-516. In: J. BARRET (Ed.). Advances in tropical aquaculture. Tahiti, vol. 9.

TAKedA, A.M.; G.Y. ShimizU \& J. HiguTI. 1990. Zoobentos de uma lagoa marginal 
(lagoa Fechada, rio Baia, Alto Paraná). Cienc. Cult. 42 (11): 1003-1007.

ThOMAZ, S.M.; F.A. LANSAC TôHA; M.C. RoBerTo; F.A. EsTEVES \& A.F. LiMA. 1992. Seasonal variation of some limnological factors of lagoa do Graraná, a várzea lake of the high rio Paraná, state of Mato Grosso do Sul, Brazil. Rev. Hydrobiol. trop. 25 (4): 269-276.

VAZzOLER, A.E.A. DE M. 1996. Biologia da reprodução de peixes teleósteos: teoria e prática. São Paulo, SBI, EDUEM, 169p.

WinEMILLER, K.O. 1987. Feeding and reproductive biology of de currito, Hoplosternum littorale, in the Venezuelan llanos with comments on the possible function of the enlarged male pectoral spines. Env. Biol. of Fishes. 20 (3): 219-227.

WootTon, R.J. 1990. Ecology of teleost fishes. London, Chapman and Hall, 404p.

Recebido em 16.VIII.1996; aceito em 13.II.1997. 